\title{
Low-cost Multimode Patch Antenna for Dual MIMO and Enhanced Localization Use
}

Adam Narbudowicz

Technological University Dublin, adam.narbudowicz@mydit.ie

Max Ammann

Technological University Dublin, max.ammann@tudublin.ie

Follow this and additional works at: https://arrow.tudublin.ie/engscheleart2

Part of the Electrical and Electronics Commons, and the Systems and Communications Commons

\section{Recommended Citation}

Narbudowicz, A. \& Ammann, M.J. "Low-cost Multimode Patch Antenna for Dual MIMO and Enhanced Localization Use", IEEE Transactions on Antenna and Propagation, vol. 66, issue 1, pp. 405 - 408, Jan. 2018. DOI: 10.1109/TAP.2017.2767643

This Article is brought to you for free and open access by the School of Electrical and Electronic Engineering at ARROW@TU Dublin. It has been accepted for inclusion in Articles by an authorized administrator of ARROW@TU Dublin. For more information, please contact arrow.admin@tudublin.ie, aisling.coyne@tudublin.ie, gerard.connolly@tudublin.ie.

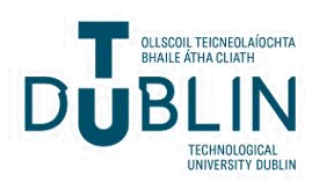




\title{
Low-cost Multimode Patch Antenna for Dual MIMO and Enhanced Localization Use
}

\author{
A. Narbudowicz and Max J. Ammann
}

\begin{abstract}
This paper proposes a simple, low-cost multi-mode patch antenna combining good MIMO performance with precise Angle of Arrival (AoA) estimation. The AoA is based on the monopulse antenna concept, however, unlike in radar applications, the necessity for complex circuitry is replaced by the intrinsic properties of even and odd resonant patch modes. This capability is advantageous for future "Internet of Things" antennas, embedded into low-cost and size-constrained devices. The envelope correlation coefficient, measured in an anechoic chamber, is below $1.5 \%$, ensuring good MIMO performance. An exemplary addition to localization algorithm exploiting antenna properties is demonstrated.
\end{abstract}

Index Terms-MIMO, localization, monopulse antenna, microstrip patch antenna

\section{INTRODUCTION}

IT is expected that the future "Smart Cities" will exploit radio communication to transform societies and boost global economy. This will require not only high data throughput between various wireless devices, but also precise localization. The former problem of high data throughput is being tackled by multiple antenna solutions, optimized for effective MIMO performance with low Envelope Correlation Coefficient (ECC). The problem of localization was extensively researched on the signal processing and algorithmic level [1 - 2] and recently by using time-modulated antenna arrays [3]. This communication proposes simple and low-cost MIMO solutions with enhanced support for localization algorithms.

Multimode patch antennas are well reported in the literature for various applications, not only for MIMO systems [4 - 6], but also for gain enhancement [7] or radiation pattern control [8-9]. It was also demonstrated, that by constructing an array of patch antennas with alternating modes, one can improve MIMO capacity performance in the case of uncertain angle of arrival [10]. These techniques rely on superposition of various patch resonant modes, which can be categorized as odd (e.g. for rectangular patch $\mathrm{TM}_{10}, \mathrm{TM}_{30}$ etc.) and even (e.g. $\mathrm{TM}_{20}, \mathrm{TM}_{40}$ etc.). Odd modes typically produce good radiation at boresight, whereas even modes produce a null at boresight. As demonstrated in this paper, this property may be applied to construct a low-cost planar monopulse system to support localization applications.

Monopulse antennas are a long standing technology, commonly used in precise long-distance radars [11-15]. They rely on two highly directive antennas (e.g. horn or leaky wave

Manuscript received September 27, 2017. This work was supported by Irish Research Council ELEVATE fellowship No. ELEVATEPD/2017/79, the European Union's Horizon 2020 programme under the Marie SklodowskaCurie grant No. 713567 and Science Foundation Ireland grant No. 13/RC/2077. antennas), placed next to each other. The output signals from the antennas are typically processed in an analogue domain to form a sum beam $\Sigma$ and a differential beam $\Delta$. This technique uses heavy directional antennas and complex processing circuitry (e.g. waveguide based Magic-T device) and up to now few implementations suitable for low-cost and compact systems were reported. An exception is [15], where a slot-loaded patch antenna with reduced size is proposed. Despite the small size, the antenna still requires a large feed network to generate the sum and differential patterns. No implementation for a localization system is discussed.

This communication investigates a previously unreported approach to integrate MIMO and monopulse systems with lowprofile, low-cost multimode patch antennas. It discusses the use of low-cost monopulse systems to complement existing localization techniques and decrease localization error due to geometric dilution of precision. The use of a multimode patch allows a reduction in the computational or circuit complexity required to generate sum and difference beams as in [3], which is key for Internet of Things applications. The low ECC demonstrates good MIMO capability and a simple localization correction algorithm is provided, demonstrating how the proposed antenna can be incorporated into a localization system.

\section{PRELIMINARY ANALYSIS}

It is well known, that a patch antenna can be approximated as a superposition of radiation from two narrow slots separated by a patch length $L_{l}$ [16]. This technique was used to provide an initial comparison between differential beams generated by a 2-element patch array (approximated as four point sources) and a single patch resonating in even-order mode (approximated as two point sources) by calculating:

$P(\phi)=\left|\frac{1}{N} \sum_{n=1}^{N} \pm \exp \left(j d_{n} k \sin (\phi)\right)\right|$

where $d_{n}$ is the distance of the $n^{\text {th }}$ point source from the coordinate origin expressed in wavelengths, $k$ represents wavenumber and the sources are located on the $y$-axis.

Assuming $\varepsilon_{\mathrm{r}}=1$, the two sources representing a single $\mathrm{TM}_{10}$ patch are separated by $0.5 \lambda$ and the sources representing $\mathrm{TM}_{40}$ patch by $2 \lambda$. A distance of $1.5 \lambda$ between the centers of two $\mathrm{TM}_{10}$ patches is assumed, so that both cases occupy the same aperture, thus allowing a fair comparison. The two peaks of the differential pattern are closer and have a steeper slope between them for the $\mathrm{TM}_{40}$ mode case, than that of the $\mathrm{TM}_{10}$ case. This

A. Narbudowicz and M. J. Ammann are with Antenna \& High Frequency Research Centre, Dublin Institute of Technology, Dublin, 8, Ireland (adam.narbudowicz@dit.ie). 
translates into greater resolution while covering a narrower angular range. The drawback of this, results in multiple sidelobes due to the distance between discrete sources being much greater than $0.5 \lambda$. This would not be acceptable for use with radar systems, where multiple beams cause ambiguity in localization. Unlike these systems however, the proposed antenna is part of a bigger localization system and is designed specifically to minimize error around boresight, which occurs due to geometric dilution of precision. With this in mind, one requires an antenna with a steep (and preferably linear for lower computational complexity) angular characteristic, where narrower angular coverage and sidelobes are of less concern. In fact, the algorithm proposed in Section VI demonstrates a

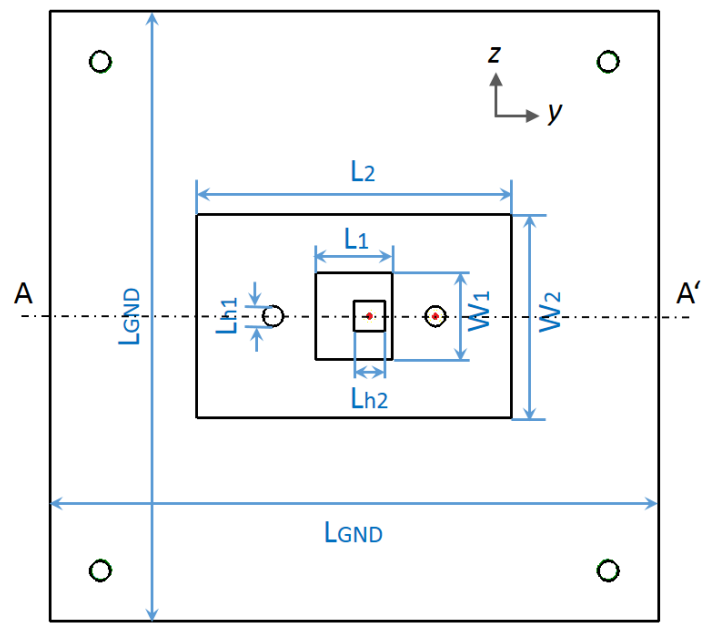

a)

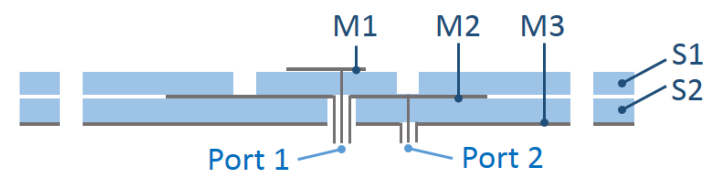

b)

Fig. 1. Antenna geometry including ground plane: a) top view; b) cut along A-A' line (layer thickness not to scale).

simple localization correction procedure that overcomes the sidelobe problem. Finally, it should be stressed that (1) is very simplified, aiming only to determine a general specification of the antenna. In real antennas the sidelobes are significantly reduced (due to the groundplane), as demonstrated in Section IV.

The calculation (1) was executed with multiple values of $\varepsilon_{\mathrm{r}}$ and also with dimensions of both realized antennas. Although the exact beamwidths change, the general conclusions remain the same for all cases. Therefore the multimode patch was selected as the main investigated design.

\section{ANTENNA DESIGN}

The proposed antenna is shown in Fig. 1. It comprises of 3 metallization layers (M1 - M3) separated by 2 layers of Taconic $^{\mathrm{TM}}$ RF-35-TC substrate (S1 - S2). Each substrate layer is $1.5 \mathrm{~mm}$ thick with $\varepsilon_{\mathrm{r}}=3.5$. The lower metallization $\mathrm{M} 3$ acts as a ground plane for the remaining layers.

The middle layer M2 comprises a patch, operating in the $\mathrm{TM}_{40}$ mode. The patch is fed through a SMA connector (port
2), with the inner pin connected to the M2 layer and outer flange to M3 layer. Since even modes produce a deep broadside null, this pattern is used for the differential beam $\Delta$. Due to greater spacing between patch edges, the $\mathrm{TM}_{40}$ mode is preferred over $\mathrm{TM}_{20}$, as it produces a steeper slope and a deeper null at boresight.

The smaller patch is located on layer M1. This is operating in $\mathrm{TM}_{10}$ mode [16], producing a single broadside beam, i.e. as the sum beam $\Sigma$. Unlike classical radar monopulse systems, the proposed antenna does not require a signal combiner (e.g. magic-T) to add and subtract signals. The outputs of two ports can be directly applied to calculate the angle of arrival, allowing simplicity and compactness of the system.

The upper patch is fed through a coaxial semi-rigid line (port 1) with the inner pin soldered to the M1 layer and the outer flange to M2 layer. This was facilitated by inserting a square slot of length $L_{h 2}=6 \mathrm{~mm}$ in layers $\mathrm{M} 3$ and $\mathrm{S} 2$ to allow soldering, which has a negligible impact on the $\mathrm{TM}_{40}$ resonant mode. Two additional circular slots of diameter $L_{h l}=4 \mathrm{~mm}$ were cut in substrate layer S1, above the inner SMA pin soldering point in M2. This is solely to avoid the presence of solder between the layers and prevent airgaps. The second slot is to preserve symmetry.

The antenna dimensions are (all in $\mathrm{mm}$ ): $L_{l}=15 ; W_{l}=17$; $L_{2}=62 ; W_{2}=40 ; L_{h 1}=4 ; L_{h 2}=6$ and $L_{G N D}=120$. Fig. 2 shows the prototype photograph.

\section{RESULTS}

Fig. 3 shows the measured and simulated (CST Microwave Studio) S-parameters. Measured bandwidths are $140 \mathrm{MHz}$ $(0.69 \%)$ for $\mathrm{S}_{11}$ and $70 \mathrm{MHz}(0.34 \%)$ for $\mathrm{S}_{22}$, which is expected for patch antennas. The significantly larger bandwidth for port 1 is due to the thicker dielectric, as the patch is located on the top-most layer M1. The isolation between the ports is greater than $35 \mathrm{~dB}$ within the antenna bandwidth.

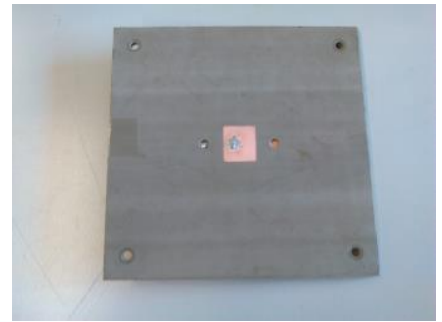

a)

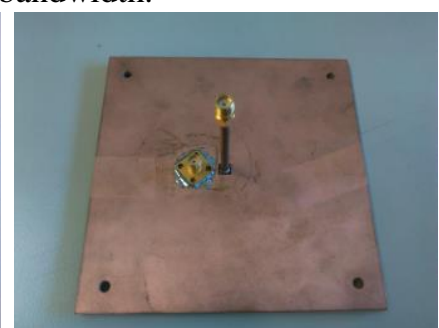

b)
Fig. 2. Prototype Photo: a) front; b) back showing feed arrangement.

Figs. $4 \mathrm{a}$ and $4 \mathrm{~b}$ show the realized gains at $5 \mathrm{GHz}$ in two principal cuts. Due to their function in the monopulse system, the pattern for port 1 is denoted as the summing beam $\Sigma$ and for port 2 as differential beam $\Delta$.The summing beam $\Sigma$ (port 1 , $\mathrm{TM}_{10}$ patch) offers a realized gain of $4.4 \mathrm{dBi}$, with a relatively broad $3 \mathrm{~dB}$ beamwidth of $125^{\circ}$ in the $x y$-plane and narrower beamwidth of only $70^{\circ}$ in the $x z$-plane (both simulation and measurement).

The differential patterns $\Delta$ show two distinctive beams in the $x y$-plane, both with similar realized gains of $6.7 \mathrm{dBi}$ and 7.4 $\mathrm{dBi}$, but with a $180^{\circ}$ phase difference. This phase difference enables differentiation between signals coming from the 
positive or negative side of the $y$-axis. The beams illuminate away from boresight, with beamwidths of $20^{\circ}$ and $19^{\circ}$.

The Envelope Correlation Coefficient was calculated by correlating the two radiation patterns $G_{1}$ and $G_{2}$ [17]:

$$
\rho_{E C C}=\frac{\iint G_{1}(\theta, \phi) G_{2}^{*}(\theta, \phi) d \Omega}{\sqrt{\iint G_{1}(\theta, \phi) G_{1}^{*}(\theta, \phi) d \Omega \iint G_{2}(\theta, \phi) G_{2}^{*}(\theta, \phi) d \Omega}}
$$

The ECC is below $1.5 \%$ throughout antenna's bandwidth, making it a very good candidate for MIMO.

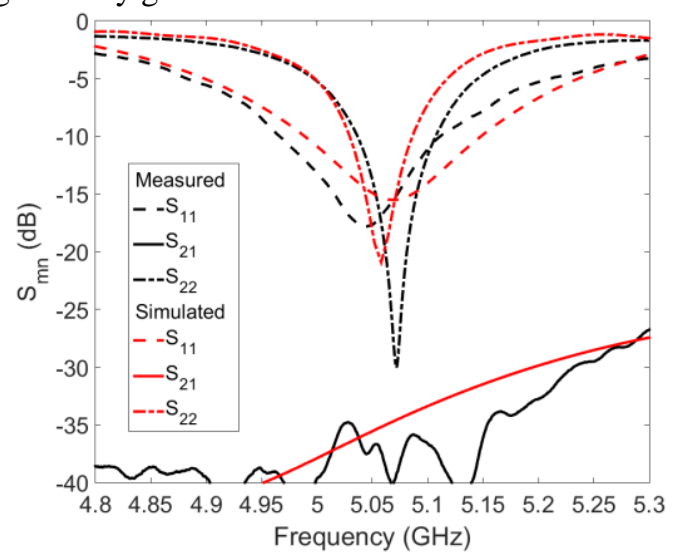

Fig. 3. Simulated and measured S-parameters of the proposed antenna

\section{COMPARISON WITH ANTENNA ARRAY}

A reference two-element antenna array was manufactured for comparison with the proposed stacked patch antenna. The centers of both patches are separated with $47 \mathrm{~mm}\left(0.78 \lambda_{0}\right)$, ensuring the same total aperture as the multi-mode antenna $\left(L_{2}\right.$ $=62 \mathrm{~mm}$ ). The array's dimensions are kept the same as the multi-mode antenna (i.e. $L_{l}=15 \mathrm{~mm}$ and $L_{G N D}=120 \mathrm{~mm}$ ), with the sole exception of $W^{\prime}{ }_{l}=14 \mathrm{~mm}$, which had to be adjusted for difference in the fringe capacitance. It should be noted that the reference array - unlike the proposed technique - requires additional circuitry to generate the sum and differential beams. To avoid the impact of this circuitry on antenna performance, the radiation patterns were measured at each port separately and added/subtracted in the post-processing step, thus assuming perfect circuitry. This was done as the beams can be generated in numerous ways and it is outside the scope of this paper to compare them.

Fig. $4 \mathrm{c}$ depicts the simulated and measured realized gains of the reference array ( $x y$-plane), alongside patterns of the

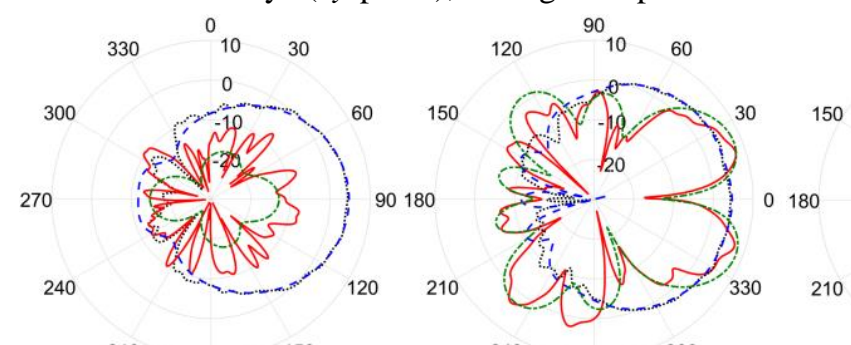

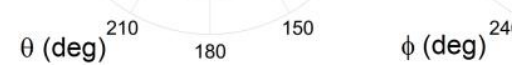

a) b) multimode antenna. Similar performance is seen, however the $\Sigma$ beam is focused more toward boresight $(8.3 \mathrm{dBi})$ and exhibits distinctive sidelobes of $-0.5 \mathrm{dBi}$ at $-60^{\circ}$ and $+0.2 \mathrm{dBi}$ at $+60^{\circ}$. The peaks of the differential beams are less pronounced (5.3 $\mathrm{dBi}$ and $5.9 \mathrm{dBi}$ ) and with a wider separation at $\phi= \pm 40^{\circ}$. This supports localization over a wider angular range, however at the cost of decreased resolution.

Fig. 4d compares the ratio of the $\Sigma$ to $\Delta$ beams for both antenna systems, which is the key feature allowing improved localization of the monopulse system. It can be seen that around boresigh (i.e. the area of primary concern) the multimode patch antenna (red) behaves more linearly and is steeper, corresponding to greater angular contrast. The angular distance between the two maxima in Fig. $4 \mathrm{~d}$ is $37^{\circ}$, thus limiting the range where linear approximation can be used to unambiguously determine the angle of arrival for localization support. Similarly, the array supports localization within a $72^{\circ}$ beamwidth, however with less linear performance.

\section{MONOPULSE LOCALIZATION CORRECTION}

Although the proposed antenna draws inspiration from classical monopulse radars, its use for localization by low-cost "smart system" nodes will significantly differ from algorithms performed in radar systems. Therefore, an exemplary localization use-case was simulated to verify the antennas localization capabilities. The monopulse capability of the proposed antenna provides increased localization accuracy in a single plane (here along $y$-axis). An additional mode can be introduced to boost localization accuracy in a second orthogonal plane, however - as the main application is seen in indoor localization systems - only localization in the $x y$-plane is analyzed. The proposed antenna acts as enabling hardware to increase localization accuracy and decreases the number of required localization nodes. The overall localization performance will also rely on the algorithms implemented and overall system design, which are both beyond the scope of this paper. Thus, the proposed study is only a representative and does not exhaust the study of possible localization algorithms appropriate for the proposed or similar antennas.

The investigated scenario (seen in Fig. 5) consists of two antennas separated by a distance of $d=20 \mathrm{~m}$ : one antenna is the proposed multimode patch with the $\Sigma$ beam directed in the $+x$ direction, whereas the second antenna can be of any type able to effectively communicate within the investigated area.

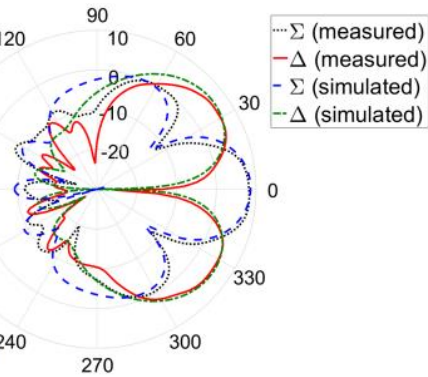

c)

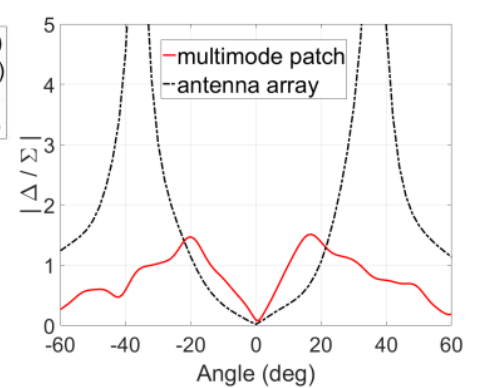

d)

Fig. 4. Measured and simulated realized gains at $5 \mathrm{GHz}$ : a) proposed antenna in $x z$-plane; b) proposed antenna in $x y$-plane; c) reference array in xy-plane; d) ratio (linear scale) of the complex differential and sum radiation patterns around boresight for both investigated antenna systems. 
A time-of-arrival approach was adopted, which measures the round-trip time of the signal to each node to pinpoint the location. An idealized scenario assumes there is no delay due to signal processing. This gives some advantages to the standard algorithm over the proposed technique. Also, it should be stated, that the time-of-arrival method was chosen solely as a representative example and the proposed correction procedure is expected to be compatible with most localization techniques.

In the investigated scenario, two problems with a standard algorithm are observed. Firstly, due to configuration symmetry, the standard algorithm is unable to distinguish whether a signal comes from the upper $(y>0)$ or lower part $(y<0)$ of the investigated area. Secondly, due to the geometric dilution of

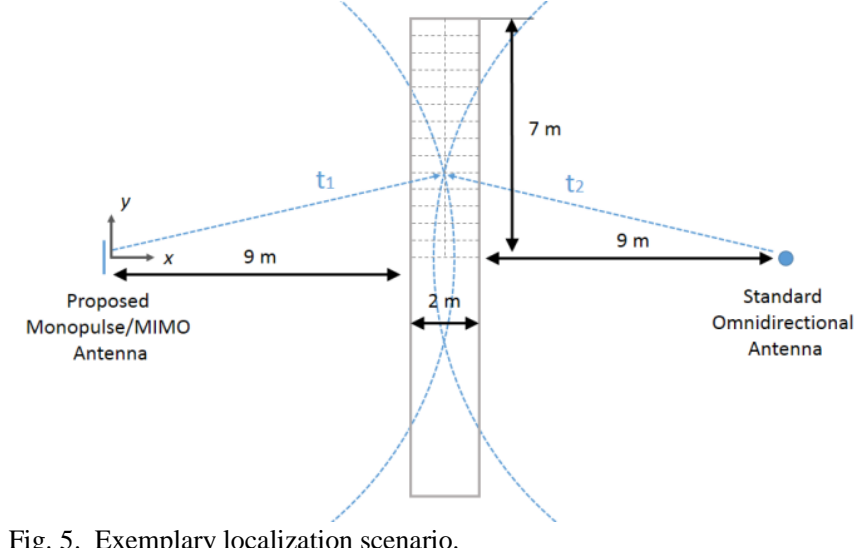

precision, the locations close to the $x$-axis will be prone to high localization error. Although both problems can be solved by adding a third antenna, this would increase the overall cost and might not be practicable for certain applications.

The proposed antenna allows the extension of the standard algorithm with a corrective procedure as follows:

1. The initial position $(x, y)$ is calculated using a standard time-of-arrival algorithm. For the investigated case

$x=\frac{t_{2}^{2}-t_{1}^{2}-d^{2}}{-2 d} ; y=\sqrt{t_{1}^{2}-x^{2}}$

where $t_{1}$ and $t_{2}$ are half round-trip times (Fig. 5).

Although a simple time-of-arrival algorithm using two nodes is implemented, it can be easily replaced by any other localization algorithm. This initial position will be corrected using monopulse signals in subsequent steps.

2. Correction for the sign of $y$ is applied:

$y=y \cdot \operatorname{sign}(\operatorname{Re}(\delta / \sigma))$

where $\delta$ is the $\Delta$ beam signal and $\sigma$ is the $\Sigma$ beam signal.

This step uses monopulse signals to determine whether the location is in the positive or negative half-plane of $y$.

3. If $\operatorname{atan}(y / x)<20^{\circ}$ another correction to $y$ is applied:

$y=x \cdot \tan (a+b \cdot \operatorname{Re}(\delta / \sigma))$

where $a$ and $b$ are coefficients of the inversed linear approximation of the function $\operatorname{Re}(\Delta(\phi) / \Sigma(\phi))$ for angles $-20^{\circ}<\phi<+20^{\circ}$, as seen in Fig. $4 \mathrm{~d}$.
This step can be applied to reduce the localization error, if the location is expected to be within the angular range covered

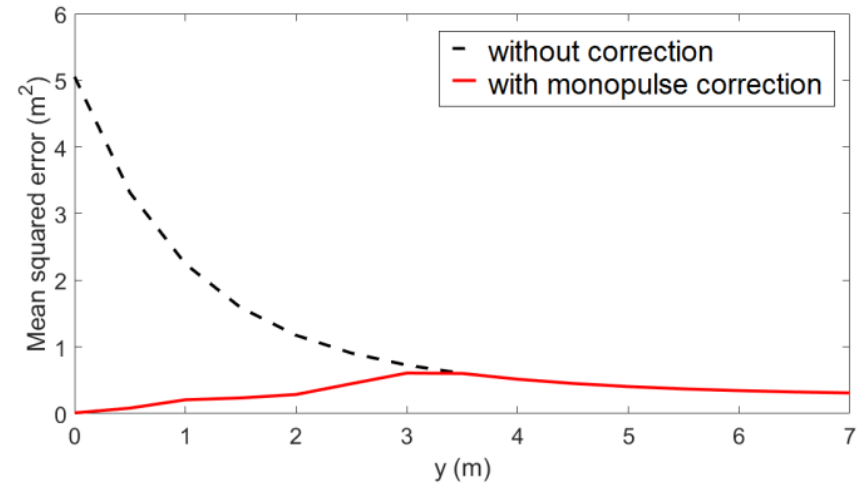

Fig. 6. MSE of $y$ for the algorithm with and without monopulse correction.

by monopulse performance, i.e. the area where the ratio between sum and differential signals can be unambiguously mapped to angle of arrival with linear approximation.

Both algorithms were tested with and without the proposed correction in an area, of $x$ from 9 to $11 \mathrm{~m}$ in $1 \mathrm{~m}$ steps and $y$ from 0 to $7 \mathrm{~m}$ in $0.5 \mathrm{~m}$ steps (seen as dashed line intersections in Fig. 5). Locations with $y<0$ were excluded from the error calculation due to the intrinsic incapability of the standard algorithm to perform well for this region. The simulation consisted of 1000 samples, where time signals $t_{1}$ and $t_{2}$ were delayed by a random number of up to $5 \%$ of their original value. Amplitudes of $\delta$ and $\sigma$ were also varied in the same manner within $\pm 10 \%$. Fig. 6 shows the Mean Squared Error (MSE) of $y$ plotted as a function of true $y$. It can be seen, that due to the geometric dilution of precision the original algorithm suffers a significant error, which decreases rapidly with increased $y$. On the other hand, the proposed correction algorithm offers very low error around boresight, which slowly increases with increased $y$. This is because step 3 [Eq. (5)] is applied only when the initial calculated position lies within the monopulse-capable beamwidth; further from boresight more samples suitable for correction might be missed due to the initial error. For increased $y$, the error of the corrected method converges to the error of the non-corrected algorithm, which is expected. The MSE for $x$ was the same for both techniques and did not exceed $0.3 \mathrm{~m}^{2}$. Overall, the proposed correction procedure allows a significant improvement for the region where the standard technique is unable to provide a reliable position, while not performing worse elsewhere.

\section{CONCLUSION}

The proposed antenna integrates MIMO capability with high precision localization of a monopulse system. Unlike bulky monopulse radar antennas, the proposed solution uses multimode patches to create sum and differential patterns, eliminating the need for complex circuitry. The antenna can be easily scaled for use with low-cost FR-4 material, significantly reducing the cost at the expense of increased losses. This makes it suitable for integration into daily-use objects, as required by smart Internet of Things applications. 


\section{REFERENCES}

[1] G. Giorgetti, A. Cidronali, S. Gupta, and G. Manes, "Single-anchor indoor localization using a switched-beam antenna", IEEE Communications Letters, vol. 13 (1), pp. 58-60, Jan. 2009.

[2] M. Rzymowski, P. Woznica, and L. Kulas, "Single-Anchor Indoor Localization Using ESPAR Antenna", IEEE Antennas and Wireless Propagation Letters, vol. 15, pp. 1183-1186, 2015.

[3] D. Masotti, "A novel time-based beamforming strategy for enhanced localization capability", IEEE Antennas and Wireless Propagation Letters, 2017. (In Press)

[4] N. Herscovici, C. Christodoulou, E. R. Iglesias, O. Q. Teruel, and M. Sanchez-Fernandez, "Compact multimode patch antennas for MIMO applications," IEEE Ant. Prop. Mag., vol. 50 (2), pp. 197-205, Apr. 2008.

[5] A. Narbudowicz, S. Koziel, M. J. Ammann, and D. Heberling, "Planar dual-mode MIMO antenna with enhanced bandwidth," in 2015 IEEE International Symposium on Antennas and Propagation \& USNC/URSI National Radio Meeting, Vancouver, BC, 2015, pp. 703-704.

[6] D. Yang, H. Zeng, R. Chen, J. Qu, Y. Wen, and S. Liu "Four port compact multimode patch antenna system for vehicular application", in 2016 IEEE Int. Workshop on Electromagnetics (iWEM), Nanjing, China, 2016.

[7] P. Juynal, and L. Shafai, "A High-Gain Single-Feed Dual-Mode Microstrip Disc Radiator," IEEE Trans. on Antennas and Propagation, vol. 64 (6), pp. 2115-2126, Jun. 2016.

[8] Z. A. Pour, and L. Shafai, "Control of Phase Center Location in Circular Microstrip Antennas operating at $\mathrm{TM}_{11}$ and $\mathrm{TM}_{02}$ modes," Microwave and Optical Technology Letters, vol. 51 (1), pp. 166-169, Jan. 2009.

[9] E. R. Iglesias, and O. Q. Teruel, "Tilting Radiation Patterns in Linear Arrays Without Phase Shifters," IEEE Trans. on Antennas and Propagation, vol. 61 (6), pp. 3360-3364, Jun. 2013.

[10] O. Q. Teruel, M. Sanchez-Fernandez, M. L. Pablo-Gonzales, and E. R. Iglesias, "Alternating Radiation Patterns to Overcome Angle-of-Arrival Uncertainty," IEEE Ant. Prop. Mag., vol. 52 (1), pp. 236-242, Feb. 2010.

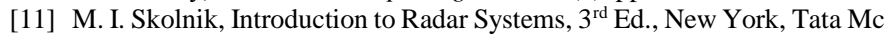
Graw Hill, 2001.

[12] J. Aliasgari, and Z. Atlasbaf, "A Novel Compact Monopulse ParallelPlate Slot Array Antenna”, IEEE Antennas and Wireless Propagation Letters, vol. 15, pp. 762-765, 2015.

[13] Zhang-Cheng Hao, Hong-Hui Wang, and Wei Hong, "A Novel Planar Reconfigurable Monopulse Antenna for Indoor Smart Wireless Access Points' Application," IEEE Trans. on Antennas and Propagation, vol. 64 (4), pp. 1250-1261, Apr. 2016.

[14] Y. H. Choun, "Sum and Tracking Radiation Patterns of a Conical Horn," IEEE Trans. on Antennas and Propagation, vol. 32 (12), pp. 1288-1291, 1984.

[15] Fei Yu, Yongjun Xie, and Z Lihua Zhang, "Single Patch Antenna With Monopulse Patterns", IEEE Microwave and Wireless Components Letters, vol. 26 (10), pp. 762-764, Oct. 2016.

[16] C. Balanis, Antenna Theory. Analysis and Design. $3^{\text {rd }}$ Ed., Hoboken, NJ, John Wiley \& Sons, 2005.

[17] M. S. Sharawi, "Current Misuses and Future Prospects for Printed Multiple-Input, Multiple-Output Antenna Systems," IEEE Antennas and Propagation Magazine, vol. 59 (2), pp. 162-170, Apr. 2017. 